\title{
Conceptos y propiedades de probabilidad en libros de texto españoles de educación primaria
}

\author{
Emilse Gómez-Torres, Universidad Nacional de Colombia (Colombia) \\ Juan J. Ortiz, Universidad de Granada (España) \\ M. Magdalena Gea, Universidad de Granada (España)
}

Recibido el 6 de julio de 2013; aceptado el 82 de Marzo de 2014

Conceptos y propiedades de probabilidad en libros de texto españoles de educación primaria

\section{Resumen}

En este artículo se analiza la presentación de conceptos y propiedades asociados a cuatro significados de la probabilidad en dos series de libros de texto españoles de educación primaria. Utilizamos una metodología cualitativa, basada en identificar y describir cómo se introduce cada concepto y propiedad en los libros de texto seleccionados. Los resultados muestran la introducción de conceptos básicos ligados a los cuatro significados, así como de propiedades fundamentales de cada uno de estos conceptos. Ambas editoriales desarrollan el significado intuitivo en todos los ciclos y el significado clásico en los dos últimos; la mención al significado subjetivo es tangencial en los dos últimos ciclos. Una diferencia notoria entre las dos series de textos es el tratamiento del significado frecuencial, una serie solo presenta conceptos y propiedades desde el punto de vista estadístico; mientras que la otra, además de éstos, desarrolla el punto de vista probabilístico. Diferencias en la forma y profundidad de tratar estos contenidos en los libros analizados resaltan la importancia del profesor al seleccionarlos y usarlos en la enseñanza.

Palabras clave: cultura estadística, alfabetización probabilística, aleatoriedad, variabilidad, incertidumbre.

\section{Conceitos e propriedades de probabilidade em textos espanhóis do ensino primário}

\section{Resumo}

Este artigo discute a apresentação de conceitos e propriedades associadas com quatro significados de probabilidade em duas séries de livros espanhóis do ensino primário. Adotamos uma metodologia qualitativa, baseada na identificação e descrição de como cada conceito e propriedade é inserida nos livros selecionados. Os resultados mostram a introdução de conceitos básicos relacionados com os quatro significados e propriedades fundamentais de cada um desses conceitos. Ambas as editoras desenvolver significado intuitivo em todos os níveis e do significado clássico nos últimos dois, a menção do sentido subjetivo é tangencial nos dois últimos ciclos. A diferença significativa entre os dois conjuntos de texto se destina frequência de tratamento, uma série só apresenta conceitos e propriedades do ponto de vista estatístico, enquanto o outro, para além destas, desenvolve ponto de vista probabilístico. As diferenças na forma e profundidade de abordar estas questões nos livros analisados destacar a importância dos professores para selecionar e usar livros no ensino.

Para citar: Gómez-Torres, E.; Ortiz, J.J. y Gea, M.M. (2014). Conceptos y propiedades de probabilidad en los libros de texto españoles de educación primaria. Avances de Investigación en Educación Matemática, $n^{\circ}$ 5, 49 - 71. 
Palavras chave: literacia estatística, alfabetização probabilística, aleatoriedade, variabilidade, incerteza.

\section{Concepts and properties of probability in primary school Spanish textbooks}

\section{Abstract}

This article discusses the presentation of concepts and properties associated with four meanings of probability in two series of Spanish primary school textbooks. We used a qualitative methodology based on identifying and describing how each concept and property is showed in the selected textbooks. The results show that basic concepts of the four meanings, and their main properties, are introduced. Both publishers develop intuitive meaning at all levels and the classical meaning in the last two, the mention of the subjective meaning is tangential in the last two cycles. A big difference between the two series of textbooks is about treatment frequency meaning, one series only presents concepts and properties from the statistical viewpoint, while the other, in addition to these, develops probabilistic viewpoint. Differences in the shape and depth of addressing these issues in the textbooks analyzed highlight the importance of teachers to select and use them in teaching.

Key words: statistical literacy, literacy probabilistic randomness, variability, uncertainty.

\section{Concepts et propriétés de probabilité dans les textes espagnols du primaire}

\section{Résumé}

Cet article traite de la présentation des concepts et des propriétés associées à quatre significations de probabilité en deux séries de manuels espagnols du primaire. Nous avons utilisé une méthodologie qualitative basée sur l'identification et la description de la manière dont chaque concept et la propriété est inscrite dans les manuels sélectionnés. Les résultats montrent l'introduction des concepts de base liés aux quatre significations et les propriétés fondamentales de chacun de ces concepts. Les deux éditeurs développent sens intuitif à tous les niveaux et la signification classique dans les deux derniers, la mention de la signification subjective est tangentielle au cours des deux derniers cycles. Une différence significative entre les deux ensembles de texte est censé fréquence de traitement, une série ne présente que des concepts et des propriétés du point de vue statistique, tandis que l'autre, en plus de ceux-ci, se développe point de vue probabiliste. Les différences dans la forme et la profondeur de ces questions dans les livres analysés en évidence l'importance des enseignants à choisir et utiliser livres dans l'enseignement.

Paroles clés: culture statistique, alphabétisation probabiliste, aléatoire, variabilité, incertitude.

\section{Introducción}

La importancia que ha adquirido la enseñanza de la probabilidad en España, desde los primeros niveles educativos, puede observarse en el decreto de enseñanzas mínimas para la educación primaria (MEC, 2006), donde en el Bloque 4. Tratamiento de la información, azar y probabilidad, se incluyen los siguientes contenidos:

Carácter aleatorio de algunas experiencias. Distinción entre lo imposible, lo seguro y aquello que es posible pero no seguro, y utilización en el lenguaje habitual, de expresiones relacionadas con la probabilidad (p. 43098; primer ciclo, niños de 6-8 años).

Valoración de los resultados de experiencias en las que interviene el azar, para apreciar que hay sucesos más o menos probables y la imposibilidad de predecir un resultado concreto. Introducción al lenguaje del azar (p. 43099; segundo ciclo, niños de 8-10 años).

Presencia del azar en la vida cotidiana. Estimación del grado de probabilidad de un suceso (p. 43101; tercer ciclo, niños de 10-12 años).

Este documento sugiere iniciar al niño en los conceptos probabilísticos elementales, mediante juegos, experimentos y observación de fenómenos naturales; para que aprenda a identificar las situaciones aleatorias y llegue, al final de la etapa, a 
asignar algunas probabilidades sencillas. Sugerencias similares se realizan en programas de otros países (e.g., NCTM, 2000).

Al considerar la enseñanza de la probabilidad hemos de tener en cuenta la forma en que se introducen los conceptos básicos y sus propiedades en los libros de texto que usa el niño; ya que muchas decisiones de los profesores, sobre las tareas a realizar con los niños, están mediadas por ellos (Stylianides, 2009). Cordero y Flores (2007) indican que determinan con frecuencia el discurso matemático escolar; mientras, que, en la enseñanza de la estadística y probabilidad, el libro contribuye a la formación del propio docente (García Alonso, 2011). Por otro lado, puesto que la probabilidad se puede presentar desde diferentes significados (Batanero \& Díaz, 2007), también es importante analizar si los libros de texto los tienen en cuenta de una forma equilibrada y adecuada a la edad del niño.

En este trabajo analizamos la introducción de los conceptos y propiedades de la probabilidad en dos series completas de libros de texto de educación primaria, con el propósito de orientar al profesor en su uso y alertarle de posibles problemas de interpretación por parte de los niños. A partir de los resultados de investigaciones previas y del análisis del currículo, se espera que nuestros resultados indiquen predominio de conceptos y propiedades ligadas al significado intuitivo de la probabilidad, una alta presencia del clásico, cierta presencia del frecuencial y baja del subjetivo.

Una vez expuesto brevemente el marco teórico y el método utilizado, se aportan los resultados obtenidos, resaltando los diferentes significados que subyacen y comparando las dos series en cada ciclo de la educación primaria. Se finaliza con unas implicaciones para la enseñanza y la formación de profesores.

\section{Fundamentos}

\subsection{Marco teórico}

Desde el currículo pretendido al implementado en el aula, una fase importante es el currículo escrito y la forma en que lo interpretan los profesores, a través de los libros de texto (Herbel, 2007). Estos libros son un producto del proceso de transposición didáctica (Chevallard, 1991), es decir, la adaptación del conocimiento matemático formal a conocimiento matemático para ser enseñado.

Un elemento fundamental en la construcción del conocimiento matemático son los conceptos y propiedades involucrados en la resolución de los problemas. Aunque conocimiento conceptual y procedimental son dos partes de un continuo y están relacionados, el conocimiento conceptual es flexible y más generalizable, ya que no está ligado a un tipo específico de problema; incluye la comprensión implícita o explícita de los principios de un dominio dado y sus interrelaciones (Rittle-Johnson \& Alibali, 1999; Rittle-Johnson, Siegler \& Alibali, 2001).

El aprendizaje de conceptos se desarrolla a lo largo de los años (Orton, 1990), por lo que es importante que se inicie el estudio de los mismos desde edades tempranas, siempre que el lenguaje y la forma de presentación sean adecuados a la edad del niño. Un principio fundamental para el aprendizaje de los conceptos, según Skemp (1980), es introducirlos mediante una adecuada colección de ejemplos y una adecuada secuenciación de actividades, en lugar de hacerlo mediante la definición. 
Los conceptos y sus propiedades son también fundamentales en el Enfoque Ontosemiótico (EOS) (Godino, Batanero, \& Font, 2007). Este postula que los objetos matemáticos emergen de las prácticas de un sujeto (persona o institución) al resolver problemas. Por ello, cuando se quiere caracterizar el significado de un objeto para una institución (por ejemplo, a través de un libro de texto) o para una persona, las prácticas observables son los indicadores empíricos que nos permiten esta caracterización. El sistema (institucional/personal) de prácticas de donde emerge un objeto matemático, como la probabilidad, se define como el significado institucional o personal del objeto dado, el cual se determina entre otros elementos por seis tipos de objetos matemáticos primarios: situaciones-problema, lenguaje, conceptos, propiedades, procedimientos y argumentos. Los autores también indican la presencia de posibles conflictos semióticos, entendiendo por tales "cualquier disparidad o discordancia entre los significados atribuidos a una expresión por dos sujetos (personas o instituciones)" (Godino, Batanero, \& Font, 2007, p.133).

\subsection{Significados de la probabilidad}

Nuestro análisis tiene en cuenta la diferenciación entre significados atribuidos a la probabilidad a lo largo de su historia; los relevantes para la enseñanza escolar son los siguientes (Batanero, 2005; Batanero \& Díaz, 2007):

Significado intuitivo. Las primeras ideas intuitivas sobre probabilidad surgen ligadas a las apuestas. Aunque los juegos de azar son tan antiguos como el hombre, el desarrollo de la teoría de probabilidades es relativamente reciente; una explicación es la antigua creencia en la imposibilidad de adivinar el futuro (Batanero, Henry, \& Parzysz, 2005), por el carácter mítico supuesto a los fenómenos aleatorios. Este significado intuitivo y el interés de los niños por los juegos pueden usarse en la enseñanza para introducir la noción de probabilidad. Reconociendo la impredecibilidad de los resultados, los niños pueden percibir que algunos sucesos merecen más confianza que otros, en función de su experiencia. La asignación de probabilidades, desde este significado, se puede hacer comparando la verosimilitud de sucesos con palabras del lenguaje habitual.

Significado clásico. La teoría de la probabilidad tiene su origen formal en el siglo XVII a partir de la resolución de problemas sobre la ganancia esperada en juegos de azar; en particular, en la correspondencia entre Pascal y Fermat. Formalización que culmina con la definición de probabilidad, dada por Laplace en 1816, como cociente entre el número de casos favorables a un suceso y el número de casos posibles. Esta definición ha sido criticada por ser circular e introducir un elemento subjetivo, asociado a la necesidad de juzgar la equiposibilidad de diferentes resultados. En adición, tiene utilidad limitada, al ser aplicable sólo en caso de espacios muestrales finitos formados por sucesos equiprobables (Batanero \& Díaz, 2007).

A pesar de estas deficiencias, este significado ha primado en la escuela durante muchos años; resultaba fácil calcular probabilidades simples en ejemplos de juegos con dados o monedas, que forman parte de la vida cotidiana del niño y donde puede aplicarse. Sin embargo, en la probabilidad compuesta, el cálculo se complica, pues se requiere razonamiento combinatorio, que es difícil para los estudiantes. Por otra parte, la ausencia de contraste con otros significados de la probabilidad promueve el sesgo de equiprobabilidad (Lecoutre, 1992) que es persistente con la edad. 
Significado frecuencial. El carácter objetivo de la probabilidad fue admitido tras la demostración de la primera ley de los grandes números, publicada por Bernoulli en 1713 (Batanero, Henry, \& Parzysz, 2005). Esta demostración también llevó al significado frecuencial, definiendo la probabilidad como el número hacia el cual tiende la frecuencia relativa al estabilizarse, en un gran número de ensayos repetidos en las mismas condiciones. Un problema filosófico es que este método no da el valor exacto de la probabilidad, sino sólo una estimación. Un problema práctico es que a veces es imposible contar con idénticas condiciones en la experimentación y es difícil saber el número de veces que ha de repetirse el experimento para obtener una estimación fiable. Más aún, en algunos campos del conocimiento no es aplicable, por su naturaleza, al ser sucesos irrepetibles. El significado frecuencial es adecuado en la enseñanza, porque tiene una aplicación más amplia que el clásico y conecta la estadística con la probabilidad. Además, las posibilidades actuales de simulación facilitan el tratamiento de este enfoque (Fernandes, Batanero, Contreras, \& Díaz, 2009).

Significado subjetivo. El teorema de Bayes, publicado en 1793, lleva al significado subjetivo de la probabilidad, pues permite transformar probabilidades a priori en probabilidades a posteriori, utilizando la información de los datos observados. Las probabilidades podrían entonces revisarse y pierden de este modo el carácter objetivo, pues dependen de la información disponible, por lo que serían definidas como grados de creencia personal. Este significado sigue siendo controvertido (Borovcnik, 2012), debido a la asunción explícita del componente subjetivo; a pesar de ello su aplicación es usual.

Desde el punto de vista de la enseñanza, Borovcnik señala su escasa presencia, en currículos vigentes. Aunque la probabilidad condicional y el teorema de Bayes se introducen habitualmente en la educación secundaria, este autor indica que el significado subjetivo no se suele desarrollar, a pesar que subyace en los procesos de simulación, pues los datos (números pseudo-aleatorios) provienen de una función dada. Por otra parte, Godino, Batanero y Cañizares (1987) sugieren usar en forma intuitiva este enfoque, en la educación primaria, con situaciones cotidianas del niño; se comenzaría asignando valores por parte del niño a las probabilidades, que se revisarían posteriormente con nuevas experiencias.

\subsection{Investigaciones previas}

La investigación sobre la presentación de la probabilidad en los libros de texto es muy escasa y concentrada en la educación secundaria, debido a que hasta la última década estaba ausente en la educación primaria; siendo aún menor la que se centra en la presentación de conceptos y propiedades.

Ortiz (2002) analizó el significado de conceptos probabilísticos y sus propiedades en una muestra de 11 libros de texto españoles para alumnos de 14-15 años, abarcando el período 1975-1991. En todos los libros se presentaban los significados clásico y frecuencial y, en casi todos, de manera formal; una minoría presentaba el significado subjetivo. Sus resultados indicaron que, aunque la mayoría introducía el concepto de espacio muestral y la frecuencia relativa de forma correcta, sólo algunos contemplaban los tres aspectos que subyacen en la idea de aleatoriedad, que son las propiedades de la frecuencia relativa, la probabilidad condicional o la variable aleatoria y hubo gran variabilidad en la presentación de las operaciones entre sucesos. 
El autor también ilustra algunos sesgos en los significados de la probabilidad presentados en los libros analizados e indica que el profesor debe mantener una permanente vigilancia epistemológica en el uso de textos para evitar su transmisión a los alumnos.

Azcárate y Serradó (2006) analizaron el contenido de probabilidad en cuatro series de libros de texto de educación secundaria obligatoria, los cuales establecen inicialmente sus objetivos e introducen una actividad inicial para motivar y evaluar los conocimientos previos de los estudiantes. Encuentran diferencias en el desarrollo de las unidades didácticas, pues mientras dos editoriales organizan los contenidos de forma lineal, comenzando con las nociones teóricas y con actividades fundamentalmente de aplicación, las organización en las otras dos es helicoidal, alternando nociones teóricas y actividades basadas en recursos manipulativos y trabajo cooperativo. Este estudio se completa en Serradó, Azcárate y Cardeñoso (2006), concluyendo que no se formalizan las relaciones entre resultados experimentales y valores teóricos, y que hay presencia mayoritaria del significado clásico en unas editoriales y del frecuencial en otras.

Barragués y Guisasola (2006) realizaron un estudio, centrado en determinados componentes de tipo epistemológico y didáctico, sobre el modo en que se introducen los conceptos relativos al azar y la probabilidad en una muestra de 34 libros de texto universitarios. Los resultados muestran que la mayoría de estos textos no presentan ciertos aspectos importantes del marco teórico de las matemáticas, como las diferentes interpretaciones de la probabilidad y sus relaciones o el desarrollo histórico de este concepto, que podrían ser utilizados para lograr un aprendizaje significativo por parte de los estudiantes. Destacan los autores que ninguno de estos libros de texto menciona los resultados de la investigación sobre las dificultades de aprendizaje de los conceptos y procedimientos de la teoría de la probabilidad, lo que explicaría los problemas que tienen los estudiantes para comprender la teoría de la probabilidad.

Otros trabajos analizan la presentación de conceptos estadísticos en libros de texto, que citamos por su relación con la probabilidad, aunque ésta no se explicita en tales estudios. Cobo y Batanero (2004), en una muestra de 22 textos de secundaria, aprecian que estos textos desarrollan significados parciales de la media aritmética; se da mucha más importancia a las definiciones y a su cálculo, que al estudio de sus propiedades.

García Alonso (2011) compara definiciones de conceptos de inferencia en cuatro libros de texto de segundo curso de bachillerato de ciencias sociales, con el significado en el diccionario de la Real Academia Española y en manuales universitarios. Los resultados muestran que los libros de texto, en ocasiones, aportan un significado incorrecto, y que algunos libros de texto usan el contexto cotidiano al definir un concepto, sin llegar a formalizarlas.

Para complementar los trabajos citados, analizaremos los conceptos y propiedades presentados en libros de texto españoles de educación primaria del currículo vigente. En lo que sigue se presentan el método y resultados del estudio.

\section{Metodología}

Las series de los libros de texto analizados se eligieron porque fueron los más utilizados en Andalucía en el curso 2011-2012 (se hizo una consulta vía web a la Consejería de Educación). Cada una de estas editoriales tiene uno o dos proyectos 
(series vigentes), dependiendo del ciclo. Nuestra muestra intencional está constituida por diez libros de texto (ver Anexo). Por cada editorial se incluyó un texto de primer ciclo, dos de segundo ciclo y dos de tercer ciclo, en total cinco de cada editorial, a los que se hará referencia en el artículo como Serie 1 o 2.

El análisis fue cualitativo y adapta la metodología de Cobo (2003):

1. Identificación de las páginas o los capítulos de los libros de texto donde se incluyen temas de azar o probabilidad. División del texto en párrafos independientes que se toman como unidades de análisis.

2. Fijación a priori de las siguientes variables para el análisis: conceptos y propiedades de la probabilidad en los significados intuitivo, clásico, frecuencial y subjetivo.

3. Para cada una estas variables se definen las categorías en que se puede clasificar una unidad de análisis. Estas categorías se determinan mediante sucesivas revisiones de los documentos curriculares, en modo cíclico e inductivo; teniendo en cuenta trabajos previos en el marco de EOS (Ortiz, 2002; Batanero, 2005; Batanero, Henry, \& Parzysz, 2005; Batanero \& Díaz, 2007).

4. Establecimiento de la presencia de cada una de las categorías en los libros de la muestra, a través de la comparación del contenido de estos textos con la lista de categorías elaborada en el paso 3. Si aparece alguna nueva se incluye en una nueva lista, hasta llegar al contenido de las Tablas 1 y 2.

5. Selección de ejemplos en los textos y elaboración de tablas cuya lectura facilite la obtención de conclusiones sobre la presentación de los conceptos y propiedades en estas dos series.

A continuación presentamos los conceptos y propiedades identificados, que hemos clasificado de acuerdo con el significado de la probabilidad al que correspondan.

\section{Conceptos}

En los libros utilizados aparece una amplia variedad de conceptos probabilísticos básicos, aunque se implementan con significados parciales y no se utiliza la terminología formal, ni se dan definiciones de los mismos. Estos conceptos en su conjunto incluyen la mayoría de ideas estocásticas consideradas fundamentales por Heitele (1975), quien opina que dichas ideas deben constituir una guía del curriculum de probabilidad desde la escuela primaria a la universidad. Asimismo desarrollan dos de las tres ideas fundamentales que forman parte de la alfabetización probabilística, según Gal (2005), puesto que su conocimiento es necesario para entender e interpretar afirmaciones probabilísticas: variación y aleatoriedad, que se comprenden en conjunción con las de constancia y determinismo.

\subsection{Conceptos relacionados con el significado intuitivo}

CI1. Azar y variabilidad. En ambas editoriales, el azar y la variabilidad, utilizados intuitivamente en la vida y lenguaje cotidianos, aparecen implícitos en la definición de experiencia de azar. Su presentación enfatiza la existencia de múltiples resultados, y está ligado principalmente a la ignorancia o inseguridad de obtener un resultado particular en una realización de un experimento. No se suele mencionar que el 
experimento se puede repetir en las mismas condiciones (lo que se exige desde el punto de vista matemático) y queda implícito, como corresponde a la edad del niño:

Una experiencia es de azar si conocemos los posibles resultados, pero no podemos asegurar cuál de ellos saldrá al final ([T8], p. 120).

Notamos que el experimento compuesto, en su mayoría de dos etapas simultáneas, se presenta como un caso de experiencia de azar. No se definen los experimentos compuestos de forma diferente a los experimentos simples; sin embargo, la composición del experimento es evidente, debido al número de dispositivos aleatorios utilizados en forma simultánea (Figura 1).

\section{Escribe posible, seguro o imposible.}

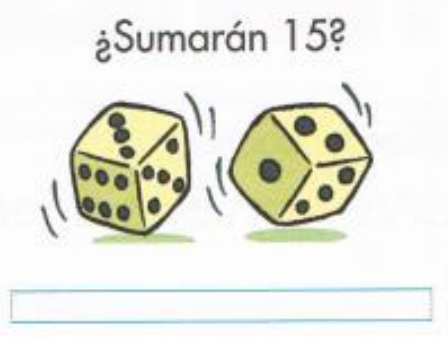

Figura 1. Experimento compuesto de dos etapas simultáneas ([T1], p. 188).

CI2. Suceso; suceso seguro, suceso posible, suceso imposible. En todos los ciclos se trabajan estas nociones. La Serie 1 introduce una definición de suceso desde segundo ciclo:

En una experiencia aleatoria, un conjunto que reúna algunos de los resultados posibles se llama suceso ([T3], p. 206)

La Serie 2 omite la definición de suceso en todos los ciclos, pero describe su tipología (seguro, imposible, posible), en función de la valoración de la posibilidad de ocurrencia (Figura 1); en consecuencia, en esta serie el concepto de suceso sólo se presenta en forma intuitiva, al igual que ocurre con los tipos de sucesos.

CI3. Posibilidad, grado de creencia. En ambas editoriales se desarrolla especialmente en el primer ciclo, y está presente en los otros dos. No se define, pero se utiliza explícitamente en las dos series cuando se pide clasificar los tipos de sucesos, como se verá en las propiedades descritas en la Sección 4.1.

\subsection{Conceptos relacionados con el significado clásico}

CC1. Juego de azar. Desde primer ciclo, la mayoría de experiencias de azar propuestas en estos textos corresponde a juegos de azar, que se basan en dispositivos aleatorios como dados, monedas y ruletas, con equiprobabilidad de los sucesos elementales. No se presenta una definición, formal o informal, en ninguna de las dos editoriales; posiblemente debido a la presencia de este tipo de juegos en la vida cotidiana del niño.

CC2. Casos favorables; casos posibles. La definición de estos conceptos queda implícita en la introducción de la regla de Laplace, en ambas editoriales. Se entiende que se denominan casos favorables a los sucesos elementales con una característica de 
interés y casos posibles a la totalidad del espacio muestral, que tampoco se define, y está descrito como "todos los resultados posibles" ([T3], p. 205) o "los resultados que se pueden obtener" ([T8], p. 217). Su introducción, mediante un ejemplo, se observa en la Figura 2.

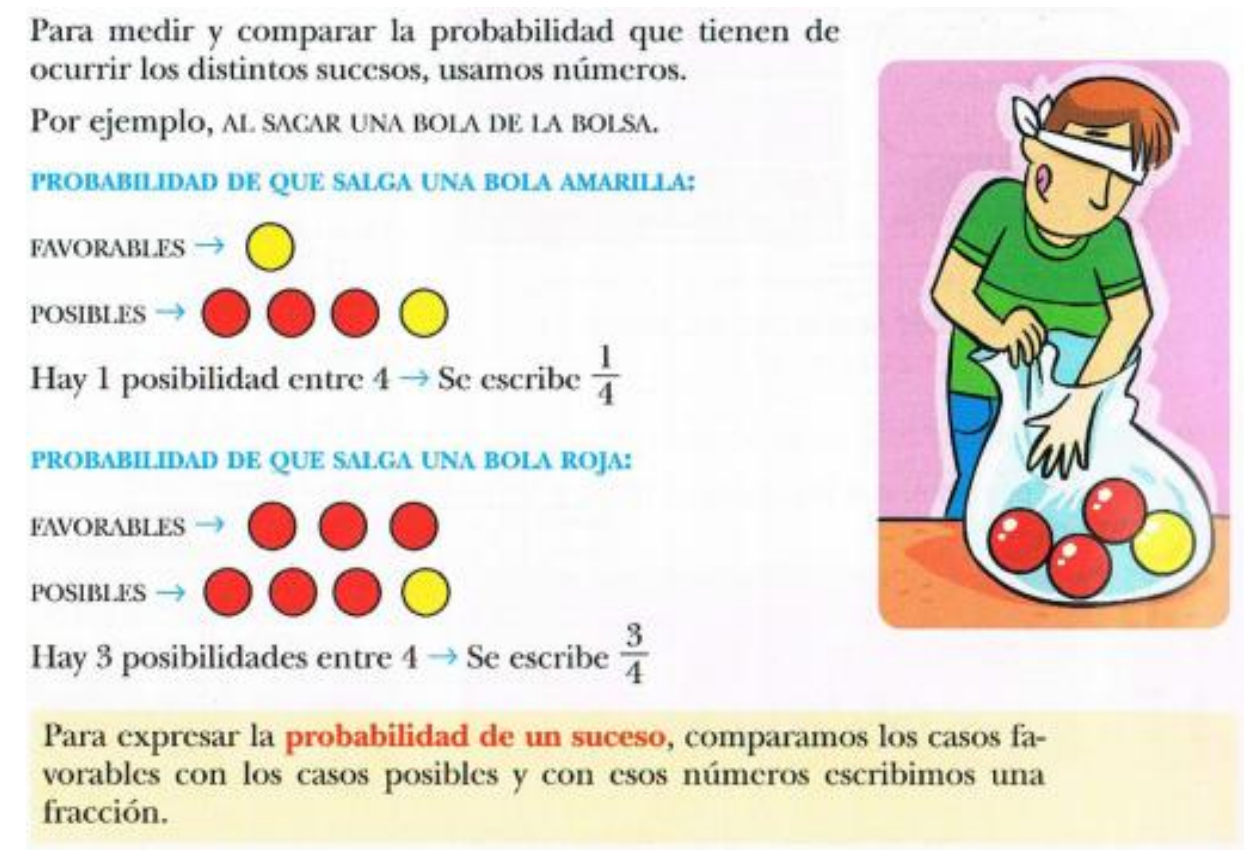

Figura 2. Probabilidad de un suceso ([T3], p. 208).

CC3. Probabilidad. En la Serie 1 se presenta en segundo ciclo como se observa en la Figura 2, y en la Serie 2 se introduce en tercer ciclo de la siguiente manera ([T10], p. 215):

La probabilidad de un suceso mide la posibilidad de que un suceso ocurra. Para calcularla utilizamos una fracción

$$
\text { Probabilidad }=\frac{\text { Número de casos favorables }}{\text { Número de casos posibles }}
$$

CC4. Juego equitativo. Este concepto apenas se menciona en los libros analizados, no se define aunque aparece en situaciones como la siguiente:

Rosa, Iria y Esteban no se ponen de acuerdo en qué película ver en el cine. Deciden lanzar dos monedas: si salen dos caras elige Rosa; si sale cara y cruz elige Iria. Si no, elige Esteban. ¿Es justo? Ayúdate de un dibujo, y explica tu respuesta. ([T9], p. 219).

\subsection{Conceptos relacionados con el significado frecuencial}

CF1. Colectivo (población). Aunque no se introduce explícitamente, este objeto matemático está presente en todas las situaciones didácticas de las páginas dedicadas a la estadística, pues los textos asumen el tratamiento de una población, que corresponde al grupo observado como totalidad de individuos.

CF2. Ensayo; ensayos repetidos. Tampoco se introduce explícitamente este objeto matemático, pero está presente en todas las situaciones didácticas de 
estadística, y en las situaciones de probabilidad donde se puede aplicar el significado frecuencial. Por ejemplo, en la Figura 3 se muestran los resultados en 10 ensayos repetidos, cada uno de ellos realizado por un mismo jugador en el lanzamiento de un tiro libre en baloncesto.

Observa el gráfico de los últimos 10 tiros libres del jugador que ves en la ilustración y estima la probabilidad que tiene de encestar el tiro que va a ejecutar.



Figura 3. Ensayos repetidos ([T5], p. 214).

CF3. Frecuencia absoluta; frecuencia relativa. Estos objetos aparecen implícitos en pocas situaciones didácticas de contenido probabilístico; su definición se asocia a estadística:

La frecuencia absoluta es el número de veces que se repite un dato. La frecuencia relativa es el cociente entre la frecuencia absoluta y el número total de datos ([T10], p. 210).

Ligado a las frecuencias, encontramos la tabla de frecuencias, que se presenta en la estadística de tercer ciclo, y sirve como precedente para la introducción de la distribución de probabilidades en la educación secundaria:

En la tabla de frecuencias organizamos los datos junto a las frecuencias que les corresponden. Si los datos son muy numerosos se agrupan en intervalos ([T4], p. 204).

CF4. Valor estimado de la probabilidad. La Serie 2 no diferencia entre probabilidad y su estimación. En la Serie 1 se desarrolla implícitamente esta diferencia en el tercer ciclo, de manera informal:

En algunas situaciones, estimamos la probabilidad atendiendo a los datos recogidos anteriormente ([T3], p. 210)

\subsection{Conceptos relacionados con el significado subjetivo}

CS1. Suceso incierto. En el significado subjetivo de la probabilidad se trabaja con sucesos inciertos, en lugar de hablar de sucesos aleatorios. En los libros analizados podemos equiparar este objeto con el de suceso posible que hemos descrito asociado al significado intuitivo (CI2). Las características que distinguen a un suceso incierto de otro determinista son inherentes a la situación en que se enmarca. Ninguna de estas editoriales menciona estas diferencias en ninguno de los tres ciclos. La Figura 4 ilustra un ejemplo de aplicación del significado subjetivo, donde podríamos entender que se presenta un suceso incierto, pues un niño de 7 años puede asignar diferentes probabilidades, dependiendo de su experiencia personal con la profesora y sus compañeros. 


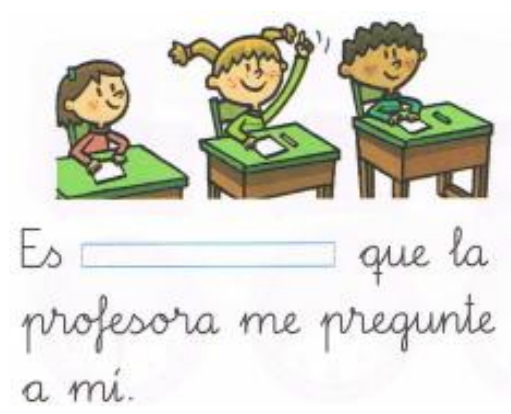

Figura 4. Situación en que se puede aplicar el significado subjetivo ([T1], p. 186).

CS2. Probabilidad como grado de creencia personal. Son poco frecuentes las situaciones ligadas al significado subjetivo, donde el niño, por su experiencia cotidiana pueda asignar una probabilidad a partir del grado de creencia personal, que puede variar de un niño a otro. En general, esta asignación no es numérica, sino a nivel del significado intuitivo (como en el ejemplo la Figura 4).

En resumen, ambas editoriales desarrollan conceptos básicos de los cuatro significados de la probabilidad, que presentamos resumidamente en la Tabla 1. En esta tabla se resalta con cursiva la idea de juego equitativo, que no aparece explícitamente como contenido en los documentos curriculares, aunque es introducido por las editoriales en tercer ciclo. Los demás conceptos están sugeridos en el Real Decreto (MEC, 2006) o por la Junta de Andalucía (Consejería de Educación, 2007).

Tabla 1. Conceptos en los libros de texto.

\begin{tabular}{|c|c|c|c|c|c|c|}
\hline \multirow{3}{*}{ Concepto según significado } & \multicolumn{2}{|c|}{$\begin{array}{l}\text { Primer } \\
\text { Ciclo }\end{array}$} & \multicolumn{2}{|c|}{$\begin{array}{l}\text { Segundo } \\
\text { ciclo }\end{array}$} & \multicolumn{2}{|c|}{$\begin{array}{l}\text { Tercer } \\
\text { ciclo }\end{array}$} \\
\hline & Serie & Serie & Serie & Serie & Serie & Serie \\
\hline & 1 & 2 & 1 & 2 & 1 & \\
\hline \multicolumn{7}{|l|}{ Intuitivo } \\
\hline CI1. Azar y variabilidad & $\mathrm{x}$ & $\mathrm{X}$ & $\mathrm{x}$ & $\mathrm{x}$ & $\mathrm{x}$ & $\mathrm{x}$ \\
\hline CI2. Suceso; seguro, posible e imposible & $\mathrm{x}$ & $\mathrm{X}$ & $\mathrm{x}$ & $\mathrm{x}$ & $\mathrm{x}$ & $\mathrm{x}$ \\
\hline CI3. Posibilidad, grado de creencia & $\mathrm{x}$ & $\mathrm{X}$ & $\mathrm{x}$ & $\mathrm{x}$ & $\mathrm{x}$ & $\mathrm{x}$ \\
\hline \multicolumn{7}{|l|}{ Clásico } \\
\hline CC1. Juego de azar & $\mathrm{x}$ & $\mathrm{X}$ & $\mathrm{x}$ & $\mathrm{x}$ & $\mathrm{x}$ & $\mathrm{x}$ \\
\hline CC2. Casos favorables; c. posibles & & & $\mathrm{x}$ & & $\mathrm{x}$ & $\mathrm{x}$ \\
\hline CC3. Probabilidad & & & $\mathrm{x}$ & & $\mathrm{x}$ & $\mathrm{x}$ \\
\hline CC4. Juego equitativo & & & & & $\mathrm{x}$ & $\mathrm{x}$ \\
\hline \multicolumn{7}{|l|}{ Frecuencial } \\
\hline CF1. Colectivo (población) & $\mathrm{x}$ & $\mathrm{X}$ & $\mathrm{x}$ & $\mathrm{x}$ & $\mathrm{x}$ & $\mathrm{x}$ \\
\hline CF2. Ensayo; ensayos repetidos & & & $\mathrm{x}$ & & $\mathrm{x}$ & \\
\hline CF3. Frecuencia (absoluta, relativa) & & & $\mathrm{x}$ & $\mathrm{x}$ & $\mathrm{x}$ & $\mathrm{x}$ \\
\hline CF4. Valor estimado de la probabilidad & & & $\mathrm{x}$ & & $\mathrm{x}$ & \\
\hline \multicolumn{7}{|l|}{ Subjetivo } \\
\hline CS1. Suceso incierto & & & $\mathrm{x}$ & $\mathrm{x}$ & $\mathrm{x}$ & $\mathrm{x}$ \\
\hline $\begin{array}{l}\text { CS2. Probabilidad como grado de } \\
\text { creencia }\end{array}$ & & & $\mathrm{x}$ & $\mathrm{x}$ & $\mathrm{x}$ & $\mathrm{x}$ \\
\hline
\end{tabular}


Se observan conceptos propios del significado intuitivo durante todos los ciclos y los significados clásico, frecuencial y subjetivo en los dos últimos ciclos. Algunos conceptos no se formalizan, como es de esperar a esta edad, solo se presentan en forma intuitiva, en especial los ligados al significado subjetivo. La Serie 2 presta mayor atención al significado clásico; mientras que la Serie 1 también desarrolla conceptos del significado frecuencial.

Hay coincidencia con el estudio de Ortiz (2002) en la mayor presencia del significado clásico y frecuencial en los textos; aunque, una de las editoriales que él analiza presenta conceptos ligados al significado frecuencial sólo desde el punto de vista estadístico, con lo que se pierde ocasión de conectar la estadística descriptiva con la probabilidad. En ambos estudios se nota la ausencia del concepto variable aleatoria y el bajo desarrollo del concepto de experimento compuesto; si bien, a nivel de primaria esta omisión es lógica por su dificultad. Este autor identifica conceptos de mayor complejidad, como son probabilidad condicional e independencia de sucesos, que corresponden a contenidos de la educación secundaria.

\section{Propiedades}

La exposición de propiedades en estos textos es informal y se implementan con significados parciales; hay pocos enunciados explícitos y ninguna demostración.

\subsection{Propiedades relacionadas con el significado intuitivo}

PII. No se puede predecir con seguridad el resultado. La impredecibilidad es la primera propiedad de los experimentos aleatorios que se introduce a los niños, por ejemplo en la actividad titulada: “QQué puede salir al lanzar un dado?” ([T6], p. 168). En los siguientes ciclos, esta pregunta se transforma en la reflexión acerca de la posibilidad de predecir un resultado para establecer si una experiencia es aleatoria.

PI2, PI3, PI4. Suceso posible (cualquier resultado de un experimento); suceso imposible (nunca se verifica); suceso seguro (siempre ocurre). Las propiedades que identifican los tres tipos de sucesos se exponen simultáneamente en ambas editoriales:

Un suceso seguro es el que ocurre siempre, un suceso posible es el que ocurre a veces, y un suceso imposible es el que no ocurre nunca. ([T8], p. 122)

Estos textos proponen actividades para que el niño identifique, o invente, sucesos de cada uno de los tres tipos en un mismo experimento aleatorio; esta situación didáctica facilita comprender que esta clasificación es excluyente y ordinal. También presentan situaciones con tres experimentos aleatorios diferentes y un mismo suceso de interés (Figura 5). Esta forma de presentación utiliza propiedades del significado axiomático no evidentes para los niños; podría llevarles a confundir la propiedad que define al suceso con una característica del dispositivo aleatorio. 


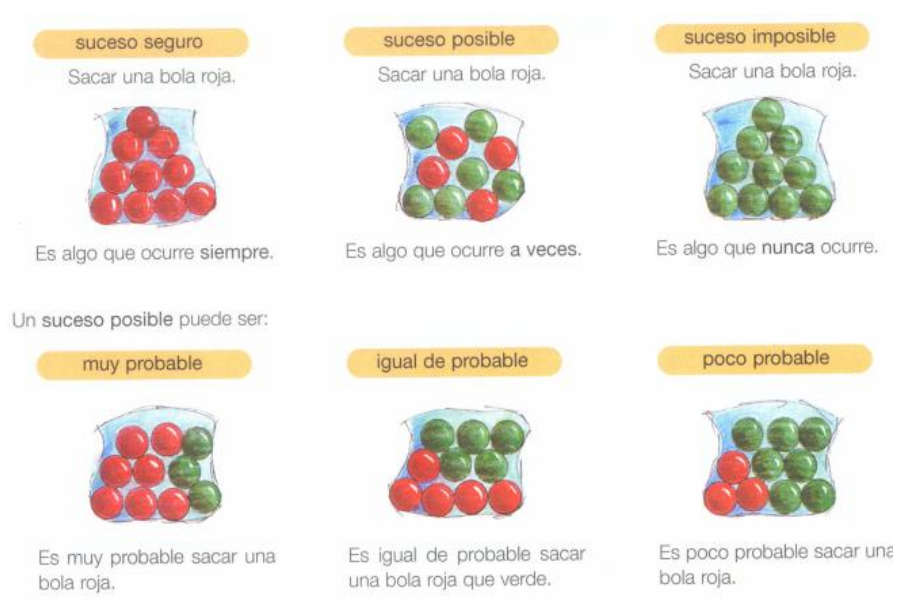

Figura 5. Tipos de sucesos y comparación de posibilidades ([T10], pp. 214).

PI5. La posibilidad de ocurrencia se puede comparar. La distinción entre sucesos según su grado de creencia sirve como introducción del concepto probabilidad, sugiere la necesidad de medirla numéricamente. La comprensión de una escala ordinal en la verosimilitud de ocurrencia de los sucesos ayudará, posteriormente, a la asignación de probabilidades con el significado subjetivo (Ortiz, 2002). La Serie 1 omite esta propiedad; mientras que la Serie 2 la presenta, en segundo y tercer ciclo. Como en los tipos de sucesos, hay dos tipos de situaciones didácticas: comparación de un mismo suceso en diferentes condiciones experimentales (Figura 5) o de varios sucesos en un mismo experimento aleatorio.

PI6. La posibilidad, como grado de creencia, se puede aproximar analizando el fenómeno. Esta propiedad vincula el significado intuitivo con los demás significados; en particular, permite analizar cuándo es más conveniente utilizar una forma u otra para la asignación de la probabilidad (Batanero, 2005). La Figura 6 ilustra esta propiedad en contraposición con la idea de suerte o con creencias de que el azar depende de condiciones externas, como la voluntad de los dioses.
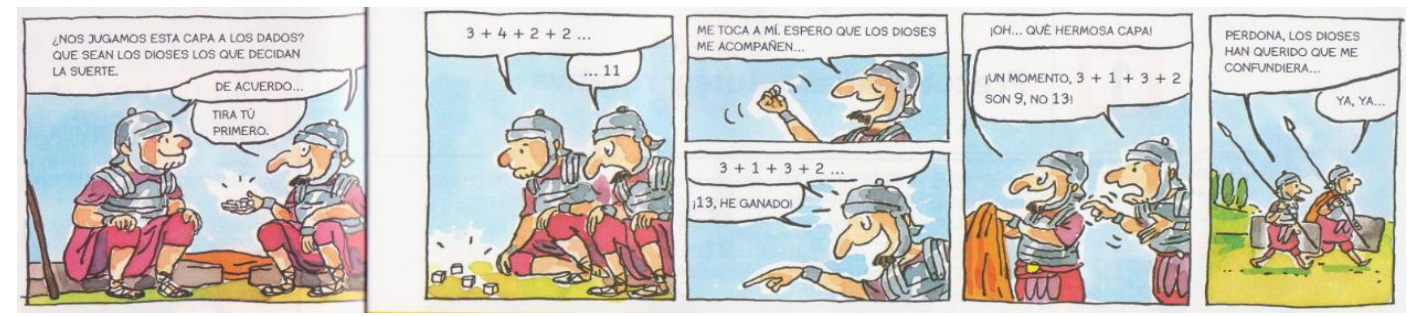

Figura 6. La posibilidad se puede aproximar analizando el fenómeno ([T10], pp. 208-209)

PI7. La posibilidad, como grado de creencia, se puede revisar con la experiencia. Esta propiedad facilita vincular el significado intuitivo con los significados frecuencial y subjetivo. A diferencia de la propiedad anterior, hace referencia al análisis de la verosimilitud en fenómenos aleatorios a partir de información previa o experiencia personal. Un ejemplo se presenta en la Figura 7. 


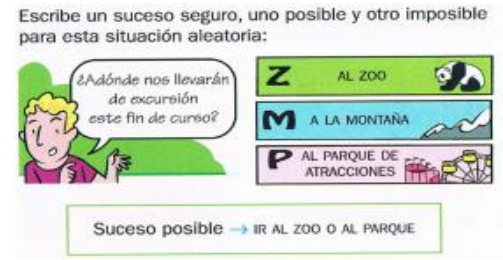

Figura 7. El grado de creencia puede cambiar con la experiencia personal ([T3], p. 207)

\subsection{Propiedades relacionadas con el significado clásico}

PC1. Número de resultados finito y numerable. Esta propiedad, que es indispensable para asignar probabilidad según este significado (Ortiz, 2002), se asume en casi todas las situaciones didácticas presentadas en estos textos. El cardinal del espacio muestral está presente en el procedimiento de cálculo, ya sea por conteo o por conocimiento previo del dispositivo aleatorio. Por ejemplo en la situación presentada en la Figura 2.

PC2. Equiprobabilidad de sucesos elementales. Se asume en la mayoría de las situaciones que se plantean en los textos, con base en la simetría física de los dispositivos aleatorios de las experiencias de azar (como ruletas con sectores de igual área). La Serie 2 menciona esta propiedad a través del siguiente ejemplo:

Si lanzamos una moneda al aire, ¿qué es más probable, sacar cara o cruz? Hay 1 cara y 1 cruz. Es igual de probable sacar cara que sacar cruz. ([T7], p. 125)

PC3, PC4. Casos favorables: resultados que favorecen; casos posibles: todos los resultados. Estas dos propiedades caracterizan los tipos de resultados que conforman los dos conjuntos de referencia para el cálculo de la probabilidad clásica. Los casos favorables se refieren a un subconjunto del espacio muestral, cuyo cardinal será el numerador de la fracción; los casos posibles hacen referencia al espacio muestral, cuyo cardinal será el denominador. Se utiliza en todas las situaciones de cálculo o comparación de probabilidades (por ejemplo en la Figura 2); sin embargo, en ningún texto se encuentra una descripción verbal de esta propiedad.

PC5. Probabilidad: Valor objetivo, calculable. Esta propiedad es válida en el contexto de juegos de azar, donde la probabilidad sólo depende del juego y del número de resultados; constituye una de las diferencias epistemológicas entre el significado clásico y los demás significados (Batanero, 2005). Un ejemplo de esta propiedad, aplicada a un experimento compuesto, se observa en la Figura 8. Es una de las situaciones más complejas para los niños, porque implica principios de combinatoria para la enumeración del espacio muestral y reconocer la ausencia de equiprobabilidad en los resultados del total a pesar de la equiprobabilidad en los experimentos simples que componen la experiencia de azar. En este ejemplo también está implícita la noción de variable aleatoria, aunque no se formaliza.

PC6. Regla de Laplace. Constituye uno de los ejes centrales del significado clásico y se utiliza sin nombrarla como tal (por ejemplo en la Figura 2). Su validez está ligada al cumplimiento de las propiedades anteriores, hecho que se omite o apenas se menciona en los textos analizados, lo que podría favorecer la aparición en los niños del sesgo de equiprobabilidad (Lecoutre, 1992). 


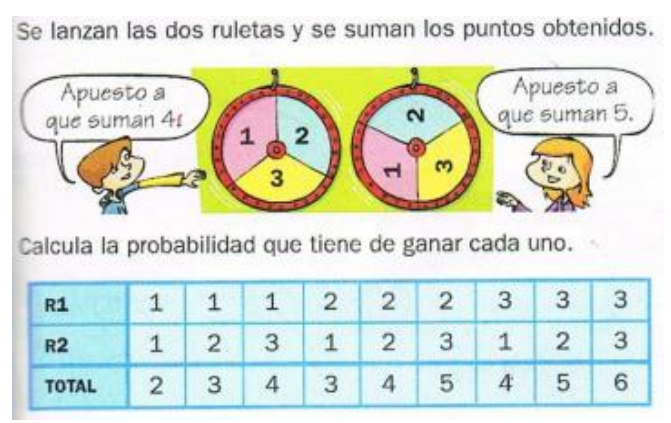

Figura 8. La probabilidad es un valor objetivo, calculable ([T5], p. 209)

PC7. La posibilidad de ocurrencia de un suceso es cuantificable. Esta propiedad es básica para llegar a la definición de función de probabilidad y vincula el significado clásico con el intuitivo. Aceptarla es un paso importante en el desarrollo del razonamiento probabilístico de los niños. Se utiliza en todas las situaciones de cálculo de probabilidades y se enuncia al introducir este procedimiento:

La probabilidad de un suceso mide la posibilidad de que ese suceso ocurra ([T10], p. 215).

\subsection{Propiedades relacionadas con el significado frecuencial}

Las siguientes propiedades están presentes en los textos analizados, de forma implícita o explícita, pero se vinculan más a estadística descriptiva que a probabilidad.

PF1. Colectivo: acontecimientos o procesos semejantes que difieren en atributos observables. Esta propiedad caracteriza las situaciones en las que aplica este significado, y hace explícito su vínculo con la estadística descriptiva. En el siguiente ejemplo se caracteriza el colectivo, en tiempo y espacio, y se resalta el atributo observable de interés en cada elemento, el color de cada coche:

Rafael ha anotado los colores de los coches que han pasado por la calle en los últimos cinco minutos. Estima la probabilidad de que el próximo coche sea blanco.

([T5], p. 211)

PF2. Los atributos en un colectivo pueden o no ser equiprobables. Casi todas las situaciones que desarrollan el significado frecuencial consideran experimentos aleatorios con resultados no equiprobables; la mayoría en contextos donde el niño puede reconocer esta propiedad (como en la Figura 9), lo que puede evitar el surgimiento del sesgo de equiprobabilidad. El libro no lo sugiere explícitamente, queda a criterio del profesor la mención de esta característica y la inconveniencia de aplicar la asignación clásica.

PF3. Probabilidad: valor objetivo, hipotético, desconocido. El carácter objetivo pero desconocido de la probabilidad distingue al significado frecuencial del clásico, aunque no se resalta explícitamente en los textos. En la Serie 1 se proponen experimentos aleatorios con repeticiones pidiendo la estimación, entendida como aproximación de la probabilidad, sin enfatizar que este valor es hipotético:

En algunas situaciones, estimamos la probabilidad atendiendo a los datos recogidos anteriormente ([T3], p. 210). 




Figura 9. Atributos no equiprobables en un colectivo ([T5], p. 211)

PF4. Aumento en la fiabilidad de la estimación con el tamaño de muestra: Esta propiedad es básica en la comprensión de la ley de los grandes números, así como en el reconocimiento de las nociones de variabilidad y precisión. La noción de límite es desconocida para los niños de estas edades, por tanto el tratamiento de esta aproximación debe hacerse en forma intuitiva, para lo cual es muy útil recurrir a la simulación y apoyo tecnológico. Las situaciones de la Serie 1 no la aplican, pues los tamaños de muestra que utilizan son muy pequeños, lo que puede promover en el niño la heurística de representatividad (Tversky \& Kahneman, 1974). Un ejemplo es el siguiente

Observa también que este tipo de estimaciones son más fiables cuantos más datos entren en la estadística; en este caso, cuántos más partidos contabilicemos ([T5], p. 210).

PF5. La frecuencia relativa de un atributo tiende a estabilizarse. La apreciación de la estabilidad de las frecuencias requiere la realización de ensayos repetidos con diferentes tamaños de muestra, que no suelen proponerse en estos textos. En la Serie 1 , solo se encuentra un ejemplo relacionado con esta propiedad. Se plantea en el capítulo de estadística en términos de las frecuencias absolutas; esta actividad realizada con frecuencias relativas, también permitiría contrastar el valor teórico con el estimado, que dejaría en evidencia la calidad de la aproximación ([T5], p. 193):

Lanza un dado 30 veces, anota los resultados obtenidos, construye su tabla de frecuencias y contesta:

a) ¿Cuáles son los valores posibles al lanzar el dado?

b) ¿Qué frecuencia absoluta de cada valor has obtenido?

c) ¿Qué crees que ocurrirá con las frecuencias absolutas a medida que aumentamos el número de lanzamientos?

PF6. Las frecuencias relativas (y las distribuciones de frecuencias) varían en cada serie de $N$ ensayos: Esta propiedad facilita la comprensión del carácter aproximado de esta asignación de probabilidad y el desarrollo del razonamiento inferencial. Su reconocimiento se obtiene a través de la reflexión de las diferencias observadas, la similitud de patrones y la posibilidad de cálculo de la probabilidad teórica. Sólo identificamos una alusión a la misma (Figura 10). 


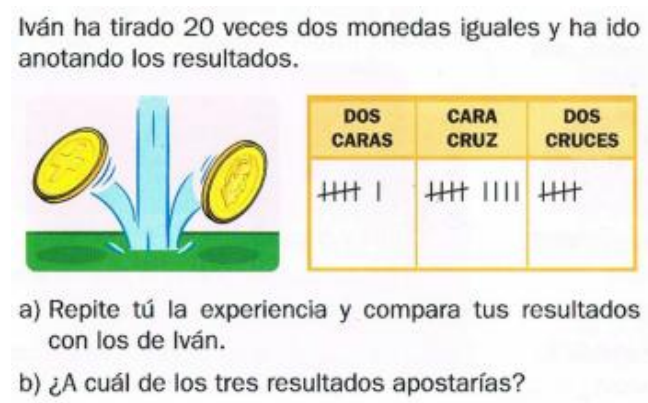

Figura 10. Variabilidad de las frecuencias en dos series de 20 ensayos ([T3], p. 211)

\subsection{Propiedades relacionadas con el significado subjetivo}

PS1. Suceso incierto: Se tiene cierta información, pero no es totalmente predecible. En el significado subjetivo se asume que la ocurrencia de un resultado está parcialmente ligada a una o varias causas, no deterministas. El cambio en la probabilidad de ocurrencia debido al conocimiento de información adicional, en relación con estas causas, no se menciona en ninguno de los tres ciclos, aunque está presente en algunas situaciones; por ejemplo cuando se pregunta “¿Cuántas rosas dará el rosal esta primavera?" ([T5], p. 204).

PS2. Probabilidad como grado de creencia: condicionada por un sistema de conocimientos. Los conocimientos sobre el fenómeno de interés permiten hacer una mejor asignación de probabilidades. Se encontraron algunos ejemplos donde aparece implícita esta propiedad, que es comprensible para los niños cuando se piensa en contextos cotidianos de toma de decisiones personales o familiares. Un ejemplo se muestra a continuación:

Posibilidades para las vacaciones:

Ya estamos como todos los años, negociando las vacaciones. Al principio pensábamos ir a París, porque allí hay un parque de atracciones fabuloso y mi madre quiere que visitemos también el museo del Louvre. ¡Imposible! Este año no llega el presupuesto, ¡otra vez será!

Descartando París, todos preferimos la playa. Así que sobre eso no hay discusión: iremos al mar con toda seguridad. Mi padre quiere alquilar un apartamento, que es más independiente. Pero mi madre prefiere un hotel, que te lo dan todo hecho. Dice que, si no, no para de trabajar. Pero que conste que en casa colaboramos todos, ¿eh?

Nosotros preferimos ir al camping. No sales del agua en todo el día y es más fácil encontrar amigos. Y, además, prometemos ayudar en todo. No sé en qué acabará la cosa. Lo más probable es que vayamos a un hotel. Pero nunca se sabe, todo es posible... ([T3], p. 20).

PS3. La probabilidad a priori es la probabilidad en ausencia de información. En este significado la ocurrencia de un resultado depende parcialmente de causas no deterministas, donde las probabilidades se denominan a posteriori cuando se ajustan a partir de la experiencia, y a priori cuando se desconocen tanto las causas como el resultado obtenido. Una mención indirecta de esta propiedad, compleja incluso para adultos, se encuentra en el siguiente ejemplo, donde la asignación clásica de probabilidades puede no ser adecuada. Por tanto, tratar solo la aproximación clásica puede promover en ellos el sesgo de equiprobabilidad ([T10], p. 209): 
La probabilidad de que salgas delegado en una clase de 30 alumnos sería de 1 entre 30 .

En la Tabla 2 se presenta un resumen de las propiedades identificadas en los textos, resaltando con cursiva las propiedades consideradas por las editoriales que no aparecen citadas en los documentos curriculares. Vemos que ambas editoriales incluyen propiedades de los cuatro significados. Se observa la presencia durante todos los ciclos de propiedades del significado intuitivo, con respecto a la impredecibilidad y a la tipología de sucesos. Las propiedades fundamentales de los significados clásico y frecuencial se presentan gradualmente; con mayor presencia del primero y muy baja atención a la experimentación y a la simulación, a pesar de la importancia que otorga el currículo de Andalucía al uso de tecnologías (Consejería de Educación, 2007). Asimismo, las dos propiedades fundamentales del significado subjetivo, sugeridas en los documentos curriculares, están mencionadas en los dos últimos ciclos.

Tabla 2. Propiedades presentes en los libros de texto.

\begin{tabular}{|c|c|c|c|c|c|c|}
\hline \multirow[b]{2}{*}{ Propiedad según significado } & \multicolumn{2}{|c|}{ Primer ciclo } & \multicolumn{2}{|c|}{ Segundo ciclo } & \multicolumn{2}{|c|}{ Tercer ciclo } \\
\hline & $\begin{array}{c}\text { Serie } \\
1\end{array}$ & $\begin{array}{l}\text { Serie } \\
2\end{array}$ & $\begin{array}{c}\text { Serie } \\
1\end{array}$ & $\begin{array}{l}\text { Serie } \\
2\end{array}$ & $\begin{array}{c}\text { Serie } \\
1\end{array}$ & $\begin{array}{l}\text { Serie } \\
2\end{array}$ \\
\hline \multicolumn{7}{|l|}{ Intuitivo } \\
\hline PI1. Impredecibilidad del resultado & $\mathrm{x}$ & $\mathrm{X}$ & $\mathrm{x}$ & $\mathrm{x}$ & $\mathrm{x}$ & $\mathrm{x}$ \\
\hline PI2. Posible: cualquier resultado & $\mathrm{x}$ & $\mathrm{X}$ & $\mathrm{x}$ & $\mathrm{x}$ & $\mathrm{x}$ & $\mathrm{x}$ \\
\hline PI3. Imposible: nunca se verifica & $\mathrm{x}$ & $\mathrm{X}$ & $\mathrm{x}$ & $\mathrm{x}$ & $\mathrm{x}$ & $\mathrm{x}$ \\
\hline PI4. Seguro: siempre ocurre & $\mathrm{x}$ & $\mathrm{X}$ & $\mathrm{x}$ & $\mathrm{x}$ & $\mathrm{x}$ & $\mathrm{x}$ \\
\hline PI5. Calificable comparando & & & & $\mathrm{x}$ & & $\mathrm{x}$ \\
\hline $\begin{array}{l}\text { PI6. La probabilidad se puede aproximar por } \\
\text { análisis del fenómeno }\end{array}$ & $\mathrm{x}$ & $\mathrm{x}$ & $\mathrm{x}$ & $\mathrm{x}$ & $\mathrm{x}$ & $\mathrm{x}$ \\
\hline $\begin{array}{l}\text { PI7. La probabilidad se puede aproximar con } \\
\text { experiencias anteriores }\end{array}$ & $\mathrm{x}$ & $\mathrm{X}$ & $\mathrm{x}$ & $\mathrm{x}$ & $\mathrm{x}$ & $\mathrm{x}$ \\
\hline \multicolumn{7}{|l|}{ Clásico } \\
\hline PC1. Número de resultados finito y numerable & $\mathrm{x}$ & $\mathrm{X}$ & $\mathrm{x}$ & $\mathrm{x}$ & $\mathrm{x}$ & $\mathrm{x}$ \\
\hline PC2. Equiprobabilidad de sucesos elementales & $\mathrm{x}$ & $\mathrm{X}$ & $\mathrm{x}$ & $\mathrm{x}$ & $\mathrm{x}$ & $\mathrm{x}$ \\
\hline PC3, PC4. Casos favorable; posibles & $\mathrm{x}$ & $\mathrm{X}$ & $\mathrm{x}$ & $\mathrm{x}$ & $\mathrm{x}$ & $\mathrm{x}$ \\
\hline $\begin{array}{l}\text { PC5. La probabilidad es un valor objetivo, } \\
\text { calculable }\end{array}$ & & & $\mathrm{x}$ & & $\mathrm{x}$ & $\mathrm{x}$ \\
\hline PC6. Regla de Laplace & & & $\mathrm{x}$ & & $\mathrm{x}$ & $\mathrm{x}$ \\
\hline PC7. La posibilidad es cuantificable & & & $\mathrm{x}$ & & $\mathrm{x}$ & $\mathrm{x}$ \\
\hline \multicolumn{7}{|l|}{ Frecuencial } \\
\hline PF1. Colectivo & $\mathrm{x}$ & $\mathrm{X}$ & $\mathrm{x}$ & $\mathrm{x}$ & $\mathrm{x}$ & $\mathrm{x}$ \\
\hline PF2. Atributos equiprobables o no & $\mathrm{x}$ & $\mathrm{X}$ & $\mathrm{x}$ & $\mathrm{x}$ & $\mathrm{x}$ & $\mathrm{x}$ \\
\hline $\begin{array}{l}\text { PF3. La probabilidad es un valor objetivo } \\
\text { hipotético, estimable }\end{array}$ & & & $\mathrm{x}$ & & $\mathrm{x}$ & \\
\hline PF4. Fiabilidad de la estimación al aumentar $N$ & & & & & $\mathrm{x}$ & \\
\hline PF5. Tiende a estabilizarse & & & & & $\mathrm{x}$ & \\
\hline PF6. Varía en cada serie de $N$ ensayos & & & $\mathrm{x}$ & & & \\
\hline \multicolumn{7}{|l|}{ Subjetivo } \\
\hline PS1. Uso de información adicional & & & $\mathrm{x}$ & $\mathrm{x}$ & $\mathrm{x}$ & $\mathrm{x}$ \\
\hline $\begin{array}{l}\text { PS2. Condicionamiento por un sistema de } \\
\text { conocimientos }\end{array}$ & & & $\mathrm{x}$ & $\mathrm{x}$ & $\mathrm{x}$ & $\mathrm{x}$ \\
\hline PS3. Probabilidad a priori & & & & & $\mathrm{x}$ & $\mathrm{x}$ \\
\hline
\end{tabular}

En adición a las sugerencias de los documentos curriculares, ambas editoriales incluyen propiedades que vinculan el significado intuitivo con los otros significados, introducen el significado clásico como una forma de cuantificación de una posibilidad, y mencionan tangencialmente la probabilidad a priori, propia del 
significado subjetivo. En particular, la Serie 1 desarrolla tres propiedades del significado frecuencial que facilitan la comprensión de su carácter aproximado y su relación con la estadística.

Vemos que los textos incluyen la mayoría de las propiedades identificadas por Ortiz (2002) en los textos de secundaria, aunque se presentan con menor formalidad; en general, de forma implícita o a través de ejemplos. J.J. Ortiz identifica también algunas propiedades adicionales, por ejemplo la ley empírica de los grandes números, y con más formalidad, como la comprobación de propiedades básicas de la frecuencia relativa. Al igual que este autor, observamos que una de las editoriales (Serie 2) presenta pocas propiedades del significado frecuencial, y únicamente en el contexto estadístico. A diferencia de este autor, notamos algunas menciones tangenciales a propiedades del significado subjetivo que no aparecen en su análisis.

\section{Conclusiones}

En este trabajo hemos mostrado la diversidad de conceptos y propiedades relacionados con la probabilidad introducidos en las series analizadas de libros de texto de educación primaria. Ello influirá sin duda en los procesos de enseñanzaaprendizaje de las matemáticas debido a la importancia citada del aprendizaje de conceptos; además, motiva el desarrollo de la alfabetización probabilística mediante el tratamiento inicial de cuatro componentes de conocimiento, de los cinco descritos por Gal (2005): ideas probabilísticas fundamentales, asignación de probabilidades y evaluación de la calidad de la información disponible, terminología y capacidad de contextualizar.

Se confirma nuestra hipótesis con respecto a la presencia de los cuatro significados de la probabilidad. En ambas editoriales, el significado intuitivo de la probabilidad se presenta desde el primer ciclo, y el significado clásico desde el segundo ciclo. El significado frecuencial de la probabilidad solo se trata en la Serie 1, principalmente en los dos últimos ciclos; si bien, ambas series exponen conceptos y propiedades ligados a este significado desde el punto de vista estadístico. En general, ambas editoriales desarrollan las propiedades de los diferentes significados sugeridas en las orientaciones curriculares, con menos presencia las del significado frecuencial, escasa atención a la experimentación y nula a la simulación (que puede desarrollarse con otro tipo de recursos que no analizamos en el presente estudio como material de multimedia). Asimismo, se presentan propiedades que no aparecen en los documentos curriculares y que han sido consideradas por las editoriales, en particular en la Serie 1.

Nuestro análisis sugiere que la presentación de la probabilidad en los textos podría conducir a un uso diferenciado de los significados de la probabilidad, dependiendo de la editorial y el ciclo educativo. También sugiere la necesidad de mejorar la formación de los profesores en la probabilidad y su didáctica; en trabajos previos (como en Contreras, Díaz, Batanero \& Ortiz, 2010) presentamos situaciones orientadas a la preparación de los profesores en estos dos aspectos complementarios.

Todos estos resultados han de interpretarse con precaución, pues, de acuerdo a Lowe y Pimm (1996), el impacto del libro de texto depende del lector y del profesor, no sólo del mismo libro, así como de las interacciones que determinan su uso en el aula. En este sentido, el profesor debe buscar estrategias para que los estudiantes progresen desde un enfoque intuitivo a otros enfoques más formales de la probabilidad, que les permita conocer los diferentes significados de este concepto. 
Ello requiere que los profesores presenten los conceptos y propiedades a través de situaciones contextualizadas y adecuadas al nivel educativo donde se enseñan.

Como reflexión final se destaca el importante papel de los autores de libros de texto, que marcan un nivel en la transposición didáctica del tema, al fijar y concretar lo establecido en los diseños curriculares. Todo ello sin descuidar la importancia del papel del profesor quien, en el aula, decide no sólo el libro de texto que recomienda a sus alumnos, sino también las partes de éste a usar en la enseñanza, así como los recursos con que debe ser complementado. Esperamos con este trabajo contribuir a la mejora de la enseñanza de las matemáticas, en particular de la probabilidad, en la educación primaria, así como facilitar la labor del profesorado en el aula.

Agradecimientos. Proyecto EDU2010-14947, FPI-BES-2011-044684 (MICINNFEDER) y grupo FQM126 (Junta de Andalucía).

\section{Referencias}

Azcárate, P., \& Serradó, A. (2006). Tendencias didácticas en los libros de texto de matemáticas para la ESO. Revista de Educación, 340, 341-378.

Barragués, J. I., \& Guisasola, J. (2006). La introducción de los conceptos relativos al azar y la probabilidad en libros de texto universitarios. Enseñanza de las Ciencias, 24(2), 241-256.

Batanero, C. (2005). Significados de la probabilidad en la educación secundaria. Revista Latinoamericana de Matemática Educativa, 8(3), 247-264.

Batanero, C., \& Díaz, C. (2007). Meaning and understanding of mathematics. The case of probability. En J.P Van Bendegen \& K. François (Eds.), Philosophical dimmensions in mathematics education. (pp. 107-127). Nueva York: Springer.

Batanero, C., Henry, M., \& Parzysz, B. (2005). The nature of chance and probability. En G. Jones (Ed.), Exploring probability in school: Challenges for teaching and learning (pp. 15-37). Nueva York: Springer.

Borovcnik, M. (2012). Multiple perspectives on the concept of conditional probability. Avances de Investigación en Didáctica de la Matemática, 2, 5-27.

Cobo, B. (2003). Significado de las medidas de posición central para los estudiantes de secundaria (Tesis doctoral no publicada). Universidad de Granada, España.

Cobo, B., \& Batanero, C. (2004). Significados de la media en los libros de texto de secundaria. Enseñanza de las Ciencias, 22(1), 5-18.

Consejería de Educación. Junta de Andalucía (2007). ORDEN de 10 de agosto de 2007, por la que se desarrolla el currículo correspondiente a la educación primaria en Andalucía.

Contreras, J. M., Díaz, C., Batanero, C., \& Ortiz, J. J. (2010). Razonamiento probabilístico de profesores y su evolución en un taller formativo. Educaçao Matemática e Pesquisa, 12(2), 181-198.

Cordero, F., \& Flores, R. (2007). El uso de las gráficas en el discurso matemático escolar. Un estudio socio epistemológico en el nivel básico a través de los libros 
de texto. Revista Latinoamericana de Investigación en Matemática Educativa, 10(1), 7-38.

Chevallard, Y. (1991). La transposición didáctica. Del saber sabio al saber enseñado. Buenos Aires: Aique.

Fernandes, J. A., Batanero, C., Contreras, J. M., \& Díaz, C. (2009). A simulação em Probabilidades e Estatística: potencialidades e limitações. Quadrante, XVIII (1 y 2), 161-183.

Gal, I. (2005). Towards "probability literacy" for all citizens: Building blocks and instructional dilemmas. En G. Jones (Ed.), Exploring probability in school: Challenges for teaching and learning (pp. 39-63). New York: Springer.

García Alonso, I. (2011). Análisis de los términos de Inferencia Estadística en Bachillerato. Números. Revista de Didáctica de las Matemáticas, 77, 51-73.

Godino, J. D., Batanero, C., \& Cañizares, M. J. (1987). Azar y probabilidad. Fundamentos didácticos y propuestas curriculares. Madrid: Síntesis.

Godino, J. D., Batanero, C., \& Font, V. (2007). The onto-semiotic approach to research in mathematics education. ZDM. The International Journal on Mathematics Education, 39(1-2), 127-135.

Heitele, D. (1975). An Epistemological View on Fundamental Stochastic Ideas. Educational Studies in Mathematics, 6, 187-205.

Herbel, B. A. (2007). From intended curriculum to written curriculum: Examining the "voice" of a mathematics textbook. Journal for Research in Mathematics Education, 38(4), 344-369.

Lecoutre, M. P. (1992). Cognitive models and problem spaces in "purely random" situations. Educational Studies in Mathematics, 23, 557-568.

Lowe, E., \& Pimm, D. (1996). 'This is so': a text on texts. En A. Bishop, K. Clements, C. Keitel, J. Kilpatrick, \& C. Laborde (Eds.) International Handbook of Mathematics Education (pp. 371-410). Dordrecht: Kluwer.

MEC (2006). Real Decreto 1513/2006, de 7 de diciembre, por el que se establecen las enseñanzas mínimas de la educación primaria. Madrid: Boletín Oficial del Estado, $\mathrm{n}^{\circ} 293$.

National Council of Teachers of Mathematics (NCTM) (2000). Principles and Standards for School Mathematics. Reston: VA, NCTM.

Ortiz, J. J. (2002). La probabilidad en los libros de texto. Universidad de Granada.

Orton, A. (1990). Didáctica de las matemáticas. Madrid: M.E.C., \& Morata.

Rittle-Johnson, B., \& Alibali, M. W. (1999). Conceptual and procedural knowledge of mathematics: Does one lead to the other? Journal of Educational Psychology, 91(1), 175- 189

Rittle-Johnson, B., Siegler, R. S., \& Alibali, M. W. (2001). Developing conceptual understanding and procedural skill in mathematics: An iterative process, Journal of Educational Psychology, 93 (2), 343-362.

Serradó, A., Azcárate, P., \& Cardeñoso, J.M. (2006). La caracterización escolar de la noción de probabilidad en libros de texto de la ESO. Tarbiya. Revista de Investigación e Innovación Educativa, 38, 91-112. 
Skemp, R. (1980). Psicología del aprendizaje de las matemáticas. Madrid: Morata.

Stylianides, G. J. (2009). Reasoning-and-Proving in School Mathematics Textbooks. Mathematical thinking and learning, 11 (4), 258-288.

Tversky, A., \& Kahneman, D. (1974). Judgement under uncertainity: Heuristics and biases. Science, 185, 1124-1131.

Anexo: Muestra de libros de texto utilizada en el análisis:

Serie 1: Editorial Anaya:

[T1]. Pérez, E., Marsá, M., Díaz, C., Ferri, T., \& Cid, O. (2011). Matemáticas 2. Proyecto Una a una.

[T2]. Ferrero, L., Gaztelu, I., Martín, P., \& Martínez, L. (2008). Matemáticas 3. Proyecto Abre la puerta, reedición 2011.

[T3]. Ferrero, L., Gaztelu, I., Martín, P., \& Martínez, L. (2008). Matemáticas 4. Proyecto Abre la puerta, reedición 2011.

[T4]. Ferrero, L., Gaztelu, I., \& Martín, P. (2009). Matemáticas 5. Proyecto Abre la puerta.

[T5]. Ferrero, L., Gaztelu, I., \& Martín, P. (2009). Matemáticas 6. Proyecto Abre la puerta.

Serie 2: Editorial S.M.

[T6]. Labarta, P., Santaolalla, E., Ferrandíz, B., \& Galve, R. (2011). Matemáticas. $2^{o}$. Primaria. Conecta con Pupi, reedición 2012.

[T7]. Peña, M., Aranzubía, V., \& Santaolalla, E. (2008). Matemáticas $3^{o}$. Proyecto Tirolina, reedición 2011.

[T8]. Peña, M., Aranzubía, V., \& Santaolalla, E. (2008). Matemáticas 4. Proyecto Tirolina, reedición 2011.

[T9]. Peña, M., Santaolalla, E., Aranzubía, V., \& Sanz, B. (2009). Matemáticas $6^{\circ}$. Proyecto Timonel, reedición 2010.

[T10]. Aranzubía, V., Santaolalla, E., Roldán, J., \& Pérez, E. (2009). Matemáticas $6^{\circ}$. Nuevo proyecto Planeta Amigo.

\section{Referencia de los autores}

Emilse Gómez, Universidad Nacional de Colombia (Colombia). egomezt@unal.edu.co

Juan J. Ortiz, Universidad de Granada (España). jortiz@ugr.es

M. Magdalena Gea, Universidad de Granada (España).mmgea@ugr.es 


\title{
Probability concepts and properties in Spanish primary school textbooks
}

\author{
Emilse Gómez-Torres, Universidad Nacional de Colombia (Colombia) \\ Juan J. Ortiz, Universidad de Granada (España) \\ M. Magdalena Gea, Universidad de Granada (España)
}

The relevance of probability teaching has increased in the latest curricula of different countries, including Spain. These curricular guidelines suggest introducing basic probability ideas to children since the initial school levels, using games of chance, experiments and observation of daily activities. The aim is that they learn to identify random experiences and to assign simple probabilities by the end of primary education. To achieve this aim we need to take into account the way in which the basic concepts and properties are introduced in the textbook used by children, because very often, the teachers' decisions concerning homework or classroom activities are guided by textbooks.

In this paper we analyze the presentation of probability concepts and properties in two Spanish primary school textbooks series, in order to guide its use by the teachers and highlight some potential misconceptions in the books. The analysis considers the specific features of four different meanings of probability along history; namely, the intuitive, classical, frequentist and subjective meanings, which are the most relevant meanings of probability for school teaching. The sample was composed by ten textbooks from two editorials, widely used in Andalucía in the academic year 2011/2012. The methodology is qualitative, where we identify and describe the way each concept and property is presented in the books. The analysis categories were established after an in deep study of Spanish curricular guidelines, and of previous research on probability, textbooks and the epistemic analysis of the aforementioned four probability meanings.

We describe the partial meanings developed in the textbooks analysed, for each concept and property. We identify how it is introduced (using a definition or an example), and either implicitly or explicitly; analyze its progression through the three cycles; the contexts used; present some examples taken from the textbook; and highlight potential semiotic conflicts, when they exist. We also present tables summarizing the presence of concepts and properties by each meaning of probability, publisher and cycle to make the comparison easier.

The results show that both textbooks series introduce a variety of basic concepts and their main properties for each of these four meanings. Globally these concepts include the fundamental stochastic ideas; however, concepts and properties are implemented with informal language, since, in general neither concepts are defined nor is property proved. Both publishers develop intuitive meaning along the three cycles, the classical meaning and a tangential mention of the subjective meaning is tangential in the last two cycles. A difference between these two publishers concerns the frequentist meaning, which is introduced in one of them from only a statistical viewpoint, while in the other, the probabilistic viewpoint is also developed.

The differences in the way and depth in which these issues are introduced in the textbooks highlight the teachers' responsibility when selecting and using them in their teaching. Consequently, in the classroom, the teacher should look for strategies to improve the children's probabilistic ideas, and move them from an intuitive approach toward a more formal understanding of probability. This paper intends to contribute to improving teaching and learning process and developing probabilistic literacy in children. 